\title{
PRINCIPAL COFIBRATIONS IN THE CATEGORY OF SIMPLICIAL GROUPS $\left({ }^{1}\right)\left({ }^{2}\right)$
}

\author{
BY \\ NORBERT H. SCHLOMIUK
}

Introduction. The aim of this paper is to develop a theory of principal cofibre bundles which is dual in the sense of Eckmann-Hilton [3] to the theory of principal fibre bundles with group $G$.

If we take as definition of a fibration the homotopical definition of Serre or a similar definition, there is a convenient dual notion of cofibration. A cofibration in the homotopical category is essentially a pair $(X, A)$ with the homotopy extension property and it plays a very important role in homotopy theory (for extensions of mappings, definition of generalized cohomology groups, etc.). In fact one can say that cofibrations have appeared in topology before fibrations (the Borsuk homotopy extension theorem is a theorem about cofibrations). For any definition of principal fibration in the homotopical category, where the fibre is an $H$-space operating associatively up to homotopy on the total space, one can obtain a dual definition of a principal cofibration in the same category. The situation changes completely if we consider the problem in the category $\mathscr{T}$ of topological spaces and continuous maps. A group $G$ in this category is a topological group and correspondingly we have in this category the notion of principal fibre bundle with group $G$. Here the group $G$ operates freely on the space $X$, and the operation is strictly associative. We could try to define a principal cofibre bundle in $\mathscr{T}$ by formally replacing groups in $\mathscr{T}$ by cogroups in $\mathscr{T}$, associative operations by associative co-operations and reverse the arrows. This is indeed possible but does not lead to a meaningful notion. The reason for this is that a cogroup in $\mathscr{T}$ reduces to a point so that there are no nontrivial cogroups in $\mathscr{T}$. On the other hand, however, there are plenty of groups in $\mathscr{T}$ (topological groups).

The situation is no better in the category $\mathscr{S}$ of simplicial sets. Here again any cogroup reduces to a point.

We are compelled to consider the next category in algebraic homotopy theory, namely the category $\mathscr{G}$ of simplicial groups. According to a theorem of Kan [10] the functor $G: \mathscr{S} \rightarrow \mathscr{G}$ establishes a 1-1 correspondence between the homotopy classes of maps: $|K| \rightarrow|L|$ (where $|K|$ is the geometric realization of $K$ ) and the

Received by the editors December 5, 1968.

(1) This paper is based on material in the author's doctoral dissertation presented to McGill University.

(2) Part of this work was done while the author was a Fellow at the Summer Research Institute of the Canadian Mathematical Congress.

Copyright (C) 1969, American Mathematical Society 
loop-homotopy classes of homomorphisms $G K \rightarrow G L$. This means that $\mathscr{G}$ is rich enough to represent homotopy theory. On the other hand, $\mathscr{G}$ is also suitable for the development of a satisfying theory of cofibre bundles. Namely (see Theorem 1.7.2) the cogroups in $\mathscr{G}$ are of the form $F K$ where $K$ is a simplicial set and $F: \mathscr{S} \rightarrow \mathscr{G}$ is the construction $F$ of Milnor. Since $|F K| \approx \Omega S|K|$ for any $K \in \mathscr{S}$ its loop space is equivalent to a cogroup in $\mathscr{G}$. Having cogroups, we are able to define co-operations and free co-operations of a cogroup $F K$ on an object of $\mathscr{G}$. The first main result is Theorem 2.2.3 which states that any principal cofibre bundle can be viewed as a twisted free product.

In \$2.1 we consider an important example of principal cofibre bundles with cofibre $F K$. This is the principal cofibre bundle of cobase $G K$ and total space $G C K$ where $G$ is the $G$-construction of $\mathrm{Kan}$ and $C K$ is the cone over $K$. This bundle is universal (Theorem 2.4.6). Indeed, any principal cofibre bundle of cofibre $F K$ and cobase $A \in \mathscr{G}$ is induced from the universal one by a map $\phi: G K \rightarrow A$. Since any principal cofibre bundle is a twisted free product, equivalence of cofibre bundles can be interpreted in terms of equivalence of twisting functions. This yields (Theorem 2.5.6) a classification of twisting functions which can be viewed as the dual of the theorem which identifies the equivalence classes of principal fibre bundles with the first cohomology set (with nonabelian coefficients).

For maps $G K \rightarrow A$ a notion of $\mu$-homotopy is defined in $\$ 2.6$ and with this notion the homotopy classes of maps: $G K \rightarrow A$ turn out be in a 1-1 correspondence with the equivalence classes of principal cofibre bundles with cofibre $F K$ and cobase $A$. This theorem is formally dual to the classification theorem for principal fibre bundles.

The author has not yet been successful in clarifying the relation between $\mu$ homotopy and loop-homotopy; he conjectures that they are equivalent. This conjecture is supported by Theorem 2.6.4 which states that the equivalence classes of principal cofibre bundles with cobase $A$ and cofibre $F S^{n}$ are in 1-1 correspondence with $\pi_{n-1}(A)$. Our results give a certain insight into the nature of Eckmann-Hilton duality and help us to understand its partial character. For instance, it now becomes clear why the desuspension problem is completely different from the dual problem of constructing a classifying space. On the other hand it has become clear in the last few years that simplicial methods are very useful for constructing certain classifying spaces ( $B_{\mathrm{PL}}, B_{\mathrm{TOP}}$, etc.). It is hoped that the concepts of this paper will turn out to be of similar use in dual situations.

The author wishes to express his grateful appreciation to Israel Berstein for suggesting the problem and for many interesting conversations and to Peter $\mathrm{J}$. Hilton for helpful comments.

\section{Preliminaries.}

1.1. We shall denote by $\mathscr{S}$ the category of simplicial sets, by $\mathscr{G}$ the category of simplicial groups, by $\partial_{i}$ the face operators and by $s_{i}$ the degeneracy operators. A 
simplicial set $K=\left(K_{n}\right)$ satisfies the extension condition of Kan if for every collection of $n+1 n$-simplices $x_{0}, x_{1}, \ldots, x_{k-1}, x_{k+1}, \ldots, x_{n+1}$ which satisfy the compatibility conditions $\partial_{i} x_{j}=\partial_{j-1} x_{i}, i<j, i \neq k, j \neq k$ there exists an $(n+1)$-simplex $x$ such that $\partial_{i} x=x_{i}$ for $i \neq k$. A simplicial set that satisfies the extension condition of Kan is called a Kan complex. Every simplicial group satisfies the extension condition of Kan [Moore].

1.2. A reduced simplicial set is a simplicial set $K$ such that $K_{0}$ consists of only one element $k_{0}$. Let $K$ be a reduced complex and let $k_{n}=s_{0}^{n} k_{0}$. Define $G(K)_{n}$ to be the free group generated by the elements $K_{n+1}-\left\{k_{n+1}\right\}$ modulo the relations $s_{0} x=e_{n}$ for $x \in K_{n}$. If $x \in K_{n+1}, \tau(x)$ denotes the class of $x$ in $G(K)_{n} . G(K)_{n}$ is freely generated by $\tau(x), x \in K_{n+1}, x \neq s_{0} y$. To define $\partial_{i}$ and $s_{i}$ on $G(K)$ it is enough to define them on generators: $\partial_{0} \tau=\left(\tau\left(\partial_{0} x\right)^{-1}\right) \partial_{i} z, \partial_{i} \tau(x)=\tau\left(\partial_{i+1} x\right)$ if $i>0, s_{i} \tau(x)$ $=\tau\left(s_{i+1} x\right)$ if $i \geqq 0$.

The functor $G$ from the category of reduced simplicial sets to the category of simplicial groups is the algebraic correspondent of the loop-space functor.

1.3. Let $K$ be a reduced simplicial set. We define a simplicial set $C K$ as follows: The simplices of $(C K)_{n}$ are the symbols $C^{k} x, x \in K_{n-k}$ with the relations:

$$
\begin{aligned}
\quad C^{n} k_{0} & =k_{n}, & & \\
\partial_{i}\left(C^{k} x\right) & =C^{k-1} x & & \text { if } i<k, \\
\partial_{i}\left(C^{k} x\right) & =C^{k}\left(\partial_{i-k} x\right) & & \text { if } i \geqq k, \\
s_{i}\left(C^{k} x\right) & =C^{k+1} x & & \text { if } i<k, \\
s_{i}\left(C^{k} x\right) & =C^{k}\left(s_{i-k} x\right) & & \text { if } i \geqq k .
\end{aligned}
$$

The complex $C K$ is contractible and the subcomplex of elements of the form $C^{0} x$ may be identified with $K$. If we collapse in $C K$ the image of $K$ to the one point simplicial set $\left(k_{n}\right)$ we obtain the reduced suspension $S K$ over $K$. Let $S^{k} x$ be the image of $C^{k} x$ in $S K$; then $(S K)_{n}$ is formed by the symbols $S^{k} x, x \in K_{n-k}, k \geqq 1$ with the relations $S^{n} k_{0}=k_{n}$.

1.4. The construction $F K=G S K$ [14]. Denote by $F K$ the simplicial group such that $(F K)_{n}$ is freely generated by $K_{n}-\left\{k_{n}\right\} ; \partial_{i}$ and $s_{i}$ operate as in $K_{n}$ and are extended to group-homomorphisms on $(F K)_{n}$.

The correspondence $x \rightarrow \tau(S x)$ extends to an isomorphism $F K \simeq G S K$. Therefore the construction $F K$ (free simplicial group generated by $K$ ) is equivalent to $G S K$ whose topological correspondent is the loop space on the suspension of $K$.

1.5. Comultiplication and cogroups. Let $G r$ be the category of groups and group homomorphisms. For $G_{1}, G_{2} \in G r$ consider the free product $G_{1} * G_{2} \in G r$ together with the injections $i_{1}: G_{1} \rightarrow G_{1} * G_{2}, i_{2}: G_{2} \rightarrow G_{1} * G_{2}$ and retractions $r_{1}: G_{1} * G_{2}$ $\rightarrow G_{1}, r_{2}: G_{1} * G_{2} \rightarrow G_{2}$. Each element of $G_{1} * G_{2}$ can be represented uniquely as a reduced word $g_{1}^{1} g_{2}^{1} \cdots g_{1}^{n} g_{2}^{n}$, where $g_{1}^{i} \in G_{1}, g_{2}^{i} \in G_{2}, g_{1}^{i} \neq e \neq g_{2}^{i}$ ( $g_{1}^{1}$ and $g_{2}^{n}$ may be equal to $e$ ). If $G_{1}=G_{2}=G$, and $g \in G$ we shall denote by $g^{\prime}=i_{1}(g)$ the same element considered in the first factor of $G_{1} * G_{2}$ and by $g^{\prime \prime}=i_{2}(g)$ the same element considered in the second factor of $G_{1} * G_{2}$. 
The folding map $\nabla: G * G \rightarrow G$ is defined by

$$
\nabla\left(g_{1}^{\prime} g_{2}^{\prime \prime} \cdots g_{2 n-1}^{\prime} g_{2 n}^{\prime \prime}\right)=g_{1} g_{2} \cdots g_{2 n-1} g_{2 n} \text {. }
$$

Definition 1.5.1. Let $G \in G \boldsymbol{r}$. A comultiplication $\Phi$ on $G$ is a map $\Phi: G \rightarrow G * G$ such that

$$
r_{1} \Phi=r_{2} \Phi=1: G \rightarrow G .
$$

EXAMPLE 1.5.2. Let $F$ be the free group generated by the set $S=\left\langle x_{1}, \ldots, x_{n}\right\rangle$. Then any map $\Phi: S \rightarrow F * F$ extends in a unique way to a group homomorphism $\Phi: F \rightarrow F * F$. Let $\Phi\left(x_{i}\right)=x_{i}^{\prime} x_{i}^{\prime \prime}, i=1, \ldots, n$. Then $\Phi$ is a comultiplication.

Definition 1.5.3. A comultiplication $\Phi: G \rightarrow G * G$ is associative if

$$
(1 * \Phi) \circ \Phi=(\Phi * 1) \circ \Phi
$$

The comultiplication of Example 1.5.2 is associative.

Definition 1.5.4. A comultiplication $\Phi$ has an inverse $\nu: G \rightarrow G$ if

$$
\nabla \circ(\nu * 1) \circ \Phi=\nabla \circ(1 * v) \circ \Phi=\text { trivial }: G \rightarrow G \text {. }
$$

The comultiplication of Example 1.5.2 has the inverse $v\left(x_{i}\right)=x_{i}^{-1}$.

Definition 1.5.5. A cogroup $(G, \Phi, v)$ in $G r$ is an object $G \in G_{r}$ together with an associative comultiplication $\Phi$ and an inverse $\nu$. In fact $\nu$ is uniquely determined by $\Phi$.

THEOREM 1.5.6. [11]. Let $(G, \Phi, r)$ be a cogroup. Then $G$ is a free group generated by the set $S$ of elements that are primitive under $\Phi$ i.e. $\Phi(x)=x^{\prime} x^{\prime \prime}$ if $x \in S$.

Hence any cogroup $(G, \Phi, r)$ is equivalent to our Example 1.5.2.

1.6. Co-operation of a cogroup on an object.

Definition 1.6.1. Let $G \in \boldsymbol{G r}$ and let $(F, \Phi, r)$ be a cogroup in $\boldsymbol{G r}$. $F$ co-operates (associatively) on $G$ if there is a homomorphism $\Psi: G \rightarrow G * F$ such that $r_{1} \Psi$ : $G \rightarrow G$ is the identity and $(1 * \Phi) \circ \Psi=(\Psi * 1) \circ \Psi$.

EXAmple 1.6.2. Let $G=H * F$ where $(F, \Phi)$ is a cogroup in $\boldsymbol{G r}$. Then $F$ cooperates on $G$ by $\Psi: H * F \rightarrow H * F * F$, where $\Psi=1 * \Phi$.

Definition 1.6.3. Let $G \in \boldsymbol{G r},(F, \Phi)$ be a cogroup in $\boldsymbol{G r}$ and let $\Psi: G \rightarrow G * F$ be a co-operation. The subgroup $A \subset G$ such that $a \in A$ if and only if $\Psi(a)=a^{\prime} \in G * F$ is called the invariant object or co-orbit object.

Definition 1.6.4. A co-operation $\Psi$ is called free if $r_{2} \circ \Psi: G \rightarrow F$ is an epimorphism.

REMARK 1.6.5. In the Example 1.6.2, $\Psi$ is clearly free and $H \subset H * F$ is the invariant object. This example is typical for free co-operations. Namely we have the following structure theorem for free co-operation:

THEOREM 1.6.6( $\left(^{3}\right)$. Let $G \in G r,(F, \Phi)$ be a cogroup in $G r$ and let $\Psi: G \rightarrow G * F$ be a free co-operation. Let $A \subset G$ be the invariant object. Then $G=A * F$ and $\Psi=1 * \Phi: A * F \rightarrow A * F * F$.

$\left({ }^{3}\right)$ Our theorem can be deduced from [7, Theorem 5.10]. Since Theorem 5.10 attributed to R. Stark is stated in [7] without proof and no published proof of it is known to the author, we shall give in the Appendix a direct proof of Theorem 1.6.6. 
1.7. Cogroups in the category of simplicial groups. If $F$ and $G$ are in $\mathscr{G}$, then $F * G \in \mathscr{G}$ satisfies $(F * G)_{n}=F_{n} * G_{n}$ and $\partial_{i}$ and $s_{i}$ are defined in the obvious way.

Definition 1.7.1. $(F, \Phi)$ is a cogroup in $\mathscr{G}, F \in \mathscr{G}, \Phi: F \rightarrow F * F$ if $\left(F_{n}, \Phi_{n}\right)$ are cogroups in $G r$, for all $n \geqq 0$.

THEOREM 1.7.2. Let $(F, \Phi)$ be a cogroup in $\mathscr{G}$. Then there exists a subcomplex $K \subset F$ such that $F=F K$ (i.e. any cogroup is the $F$ construction over some $K$ ).

Proof. By Theorem 1.5.6, $F_{n}$ is generated freely by the set $K_{n} \subset F_{n}$ of elements such that $\Phi_{n}(x)=x^{\prime} x^{\prime \prime}$. Let $K$ denote the collection of $K_{n}$ 's $n=0,1, \ldots$ If $x \in K_{n}$, $\Psi\left(\partial_{i} x\right)=\partial_{i} \Psi(x)=\partial_{i}\left(x^{\prime} x^{\prime \prime}\right)=\partial_{i} x^{\prime} \partial_{i} x^{\prime \prime}=\left(\partial_{i} x\right)^{\prime}\left(\partial_{i} x\right)^{\prime \prime}$ so that $\partial_{i} x \in K_{n-1}$. Similarly if $x \in K_{n}, s_{i} x \in K_{n+1}$. Therefore $K$ is a complex and $F=F K$.

Definimion 1.7.3. Let $G$ be a simplicial group and let $F=(F K, \Phi)$ be a cogroup in $\mathscr{G}$. We shall say that $F$ co-operates on $G$ if there is a simplicial group map $\Psi: G \rightarrow G * \mathscr{F}$ such that:

$$
r_{1} \Psi=1: G \rightarrow G, \quad(\Psi * 1) \circ \Psi=(1 * \Phi) \circ \Psi .
$$

Definition 1.7.4. The co-operation is free if $r_{2} \Psi: G \rightarrow F$ is an epimorphism.

\section{Statement of results.}

\subsection{Principal cofibre bundles in $\mathscr{G}$.}

Definition 2.1.1. A principal cofibre bundle in $\mathscr{G}$ is a triple $A, G, F \in \mathscr{G}$ where $(F, \Phi)$ is a cogroup in $\mathscr{G}$ and $(F, \Phi)$ co-operates freely by $\Psi$ on $G, A \subset G$ being the invariant object.

We shall call $A$ the cobase, $i: A \rightarrow G$ the cofibration, $F$ the cofibre and $G$ the total object of the cofibre bundle.

MAIN EXAMPLE 2.1.2. The cofibre bundle $(G K, G C K, F K)$. For any reduced complex $K, F K$ co-operates freely on $G C K$. Since $G C K$ is generated freely by symbols $\tau\left(C^{0} x\right)=\tau(x)$ and $\tau(C x)$, to define a map $\Psi: G C K \rightarrow G C K * F K$, it is enough to define $\Psi$ on the free generators $\tau\left(C^{0} x\right)=\tau(x)$ and $\tau(C x)={ }^{1} x$. Let

$$
\Psi(\tau(x))=(\tau(x))^{\prime}, \quad \Psi\left({ }^{1} x\right)={ }^{1} x^{\prime} x^{\prime \prime} .
$$

Then $\Psi$ is a co-operation. The invariant object of the co-operation $\Psi$ is generated by the elements $\tau(x), x \in K$ hence the invariant object is $G K \subset G C K$. It can be verified that the co-operation is associative and it is clearly free. The cofibration $(G K, G C K, F K)$ is a principal cofibre bundle with cobase, cofibre and cogroup $F K$. Taking into account the topological interpretations of the terms, we see that it corresponds to the cofibration $X \rightarrow C X \rightarrow S X$ where $C X$ is the cone over $X$ and $S X$ the suspension. We shall prove that $(G K, G C K, F K)$ is a universal principal cofibre bundle with cogroup $F K$, induced by a map of $G K$ into the cobase of the universal cofibration.

2.2. Any principal cofibre bundle is a twisted free product.

Definition 2.2.1. Let $A$ and $B=F K$ be simplicial groups. $G=A *_{t} B$ is called the twisted free product of $A$ and $B$ with twisting function $t: K \rightarrow A$ if as an abstract 
group, $G_{n}=A_{n} *{ }^{1} B_{n}$ where $B_{n}$ is isomorphic to ${ }^{1} B_{n}$ (isomorphism $b \rightarrow{ }^{1} b$ ) and on $A_{n}, \partial_{i}$ and $s_{i}$ are those on $A$, whereas for $x \in K_{n}$ :

$$
\begin{aligned}
s_{i}{ }^{1} x & =s_{i} x, \quad i \geqq 0, \\
\hat{\partial}_{i}{ }^{1} x & =\partial_{i} x, \quad i>0, \\
\partial_{0}{ }^{1} x & =t(x) \partial_{0} x .
\end{aligned}
$$

This means that $G$ is almost the free product of $A$ and $B$ with a twisting in the definition of the first face operator of $B$.

EXAMPLE 2.2.2. Let ${ }^{1} F \subset G C K$ be the subgroup generated by the elements ${ }^{1} x(=\tau(C(x)))$. Then, as an abstract group $(G C K)_{n}=(G K)_{n} * F$, where ${ }^{1} F_{n}$ is isomorphic as a group to $F=F K$ and since

$$
\begin{aligned}
\partial_{0}{ }^{1} x & =\tau(x)^{-1} \partial_{0} x, \\
\partial_{i}{ }^{1} x & =\partial_{i} x, \quad i>0, \\
s_{i}{ }^{1} x & =s_{i} x, \quad i \geqq 0 .
\end{aligned}
$$

$G C K$ is the twisted free product of $G K$ and $F$, with the twisting function $t: K \rightarrow G K$ defined on the generators by: $t(x)=\tau(x)^{-1}$. We shall prove in $\S 3$ :

THEOREM 2.2.3. Let $(A, G, F)$ be a principal cofibre bundle. Then $G$ is isomorphic to the twisted free product $G=A *_{t} F$, with some twisting function $t: F \rightarrow A$.

2.3. Induced bundles. Let $(G, A, F, \Psi)$ be a principal cofibre bundle and let $\Phi: A \rightarrow B$ be a map of simplicial groups. We construct a new simplicial group $H$ as follows: $H$ is the quotient group obtained from $B * G$ by introducing the relations $\Phi(a) a^{-1}=e_{n}$ for all $a \in A_{n}$. Let $g: B \rightarrow H$ be the composition of the natural inclusion $i: B \rightarrow B * G$ and the epimorphism $\pi: B * G \rightarrow H$. $F$ cooperates on $B * G$ by co-operating on the second factor, namely $\Psi^{1}: G * G \rightarrow B * G * F$ is defined by $\Psi^{\prime \prime}=1 * \Psi$ where $\Psi: G \rightarrow G * F$ is a co-operation. If $z=\Phi(a) a^{-1}$, $a \in A \Psi(z)=(\Phi(a) a)^{\prime-1}$. Therefore if we map:

$$
\pi * 1: B * G * F \rightarrow H * F
$$

then $(\pi * L) \Psi^{\prime}(z)=\pi\left((\Phi(a))^{\prime} a^{\prime-1}\right)=e$, so that the co-operation $\Psi^{\prime}$ induces a cooperation $\Psi^{*}: H \rightarrow H * F$ such that the diagram

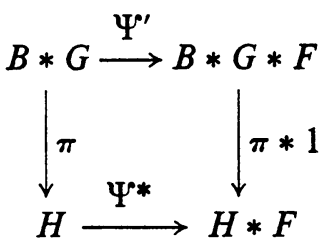

is commutative.

In the terminology of group theory, $H$ is the amalgamated free product of $B$ and $G$, the subgroup $A \subset G$ being amalgamated with its image by $\Phi$ in $B$. According to 
a theorem about such products, $G: B \rightarrow H$ is a monomorphism. We shall identify $B$ with its image in $H$. The subgroup $B \subset H$ is invariant under $\Psi^{*}$. Indeed, $\Psi^{*}(b)=\Psi^{\prime \prime}(b)=b^{\prime}$. It follows from Theorem 2.2.3 that $H$ is the twisted free product of $B$ and $G$ with the twisting function $t^{*}: K \rightarrow B, t^{*}=\Phi \circ t$. As an abstract group $G_{n}=A_{n} *{ }^{1} F$. Therefore

$$
(B * G)_{n}=B_{n} * G_{n}=B_{n} * A_{n} *{ }^{1} F_{n} .
$$

The amalgamation of $A_{n}$ and $\Phi\left(A_{n}\right)$ does not affect $F_{n}$ so that $H_{n}=B_{n} *{ }^{1} F_{n}$.

It is clear that the image under the twisting function of $x \in K_{n}$ will be the class of $t(x)$ in $B_{n}$ i.e. $\Phi t(x)$ and this completes the proof of

THEOREM 2.3.1. ( $\left.H, A, F, \Psi^{*}\right)$ is a principal cofibre bundle which may be represented as $H=B *_{\Phi} F$.

We shall call $\left(H, A, F, \Psi^{*}\right)$ a bundle induced from $(G, A, F, \Psi)$ via $\Phi$.

2.4. Universal principal cofibre bundles. Consider together with Example 2.1.2 another principal cofibre bundle $H=B *_{r} F K$, represented as a twisted free product with twisting function $r$. In $\S 4$, we shall prove:

THEOREM 2.4.1. For any principal cofibre bundle $\left(H, B, F K, \Psi^{*}\right)$ represented as a twisted free product $B *_{r} F K$ with twisting function $r: K \rightarrow B$, there exists a map (of simplicial groups) $\Phi: G K \rightarrow B$ such that $\left(H, B, F K, \Psi^{*}\right)$ is induced from (GCK, $G K$, $F K, \Psi)$ via $\Phi$ and $r=\Phi \circ t$ where $t: K \rightarrow G K$ is the canonical twisting function of the universal bundle.

2.5. The classification theorem for principal cofibre bundles.

Definition 2.5.1. Let $(G, A, F)$ and $(H, A, F)$ be two principal cofibre bundles with the same cobase $A$. A map of simplicial groups $\lambda: G \rightarrow H$ is a principal cofibre bundle equivalence if the diagram

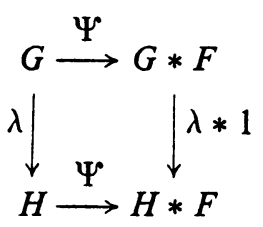

is commutative and if $\lambda \mid A=1$.

Let ${ }^{1} x$ be a primitive element i.e. $\Psi\left({ }^{1} x\right)={ }^{1} x^{\prime} x^{\prime \prime}, x$ generator of $F$. Then $\lambda\left({ }^{1} x\right)$ is also a primitive element corresponding to the generator $x$.

Let $G=A *_{s} F$ and $H=A *_{t}^{1} F$ be two twisted-product representations of the bundles $G$ and $H$ with twisting functions $s, t: K \rightarrow A$. We have by definition $G_{n}=A_{n} *{ }^{1} F_{n}$ and $H_{n}=A_{n} *{ }^{2} F_{n}$. Since $\lambda\left({ }^{1} x\right)$ is primitive, it differs by an invariant element $\mu(x)$ from the element ${ }^{2} x$, corresponding in the second representation to $x$. 
Therefore, $\lambda\left({ }^{1} x\right)=\mu(x)^{2} x$ and $\mu(x)$ completely determines $\lambda(x)$ since $\lambda$ is the identity on $A_{n}$. Let us see how $\mu$ behaves with respect to $\partial_{i}$ and $s_{i}$

so that

$$
\begin{aligned}
& s_{i} \lambda(x)=s_{i} \mu(x) s_{i}^{2}(x)=\lambda\left(s_{i} x\right)=\mu\left(s_{i} x\right) s_{i}^{2}(x), \quad i \geqq 0 \\
& \hat{c}_{i} \lambda(x)=\partial_{i} \mu(x) \partial_{i}^{2} x=\lambda\left(\partial_{i} x\right)=\mu\left(\partial_{i} x\right) \partial_{i}^{2} x, \quad i>0
\end{aligned}
$$

$$
s_{i} \lambda(x)=\mu\left(s_{i}(x)\right), \quad i>0 ; \quad \partial_{i} \mu(x)=\mu\left(\hat{o}_{i} x\right), \quad i>0
$$

Further

$$
\partial_{0} \lambda\left({ }^{1} x\right)=\partial_{0} \mu(x) t(x) \partial_{0}^{2} x=\lambda\left(\partial_{0} x\right)=\lambda(s(x)) \lambda\left(\partial_{0} x\right)=s(x) \mu\left(\partial_{0} x\right)^{2} \partial_{0} x .
$$

Therefore

$$
\partial_{0} \mu(x)=s(x) \mu\left(\partial_{0} x\right) t(x)^{-1} .
$$

Definition 2.5.5. Two twisting functions $s, t: K \rightarrow A$ are called equivalent if there exists a function $\mu: K \rightarrow A$ preserving degrees and such that (2.5.3) and (2.5.4) are satisfied.

Hence we have proved

THEOREM 2.5.6. There is a 1-1 correspondence between the equivalence classes of twisting functions $t: K \rightarrow A$ and the equivalence classes of cofibre bundles with base $A$ and cofibre $F=F K$.

This theorem is remarkably similar to the description of principal fibre bundles with base $A$ and group $G$ and the first cohomology set of $A$ with coefficients in the germs of functions $A \rightarrow G$. Here the nonabelian cohomology is replaced by some kind of nonabelian homology, the cycles being the twisting functions.

2.6. Equivalence and $\mu$-homotopy. We shall now introduce a definition of homotopy of simplicial homomorphisms $\Phi: G K \rightarrow A$. This is an equivalence relation defined only for homomorphisms whose domains are of the form $G K$. This is not unexpected since the loop-homotopy is an equivalence relation only on free simplicial groups and the $G K$ 's form the most important case of free simplicial groups. Furthermore, it probably coincides with the loop-homotopy equivalence, but so far the author has not been able to prove this statement, hence we state it as a conjecture.

Definition 2.6.1. Two simplicial homomorphisms $\Phi_{0}, \Phi_{1}: G K \rightarrow A$ are $\mu$ homotopic if there exists a map $\mu: K \rightarrow A\left(\mu\left(K_{n}\right) \subset A_{n}\right)$ such that:

$$
\begin{array}{rlrl}
\partial_{0} \mu(x) & =\Phi_{0}(\tau(x))^{-1} \mu\left(\partial_{0} x\right) \Phi_{1}(\tau(x)), \\
\partial_{i} \mu(x) & =\mu\left(\partial_{i} x\right), & i>0, \\
s_{i} \mu(x) & =\mu\left(s_{i} x\right), & i \geqq 0 .
\end{array}
$$

In view of the fact that the correspondence $t \rightarrow \Phi$ which associates to each twisting function $t: K \rightarrow A$, the simplicial group map $\Phi: G K \rightarrow A$ defined by 
$\Phi(\tau(x))=t(x)^{-1}$ is $1-1$ and in view of the above definition, the following theorem is a restatement of Theorem 2.5.6.

THEOREM 2.6.5. There is a 1-1 correspondence between the principal cofibre bundles of cofibre FK and cobase $A$ and the $\mu$-homotopy classes of maps $\Phi: G K \rightarrow A$.

Formally, at least, this is a dual of the classification theorem of principal fibre bundles.

2.7. Classification of principal cofibre bundles with a sphere as cofibre. Although apparently unsatisfactory because of the lack of identity between $\mu$-homotopy and loop-homotopy, Theorem 2.6.5 yields a complete classification of principal cofibre bundles with cofibre $F S^{n}$. Recall that $S^{n}$ is a simplicial set with one vertex $k_{0}$, one nondegenerate simplex $x$ in dimension $n$ and no nondegenerate simplexes in higher dimensions. To specify a simplicial homomorphism $\Phi: G S^{n} \rightarrow A$, it is enough to specify its value $\Phi(\tau(x))$. Furthermore we have in $G K$,

$$
\partial_{i} \tau(x)=e_{n-2}, \quad i=0, \ldots, n-1 .
$$

Therefore,

$$
\partial_{i} \Phi(\tau(x))=\Phi\left(\partial_{i} \tau(x)\right), \quad i=0, \ldots, n-1 .
$$

We have proved that the simplicial homomorphisms: $G S^{n} \rightarrow A$, are in 1-1 correspondence with elements $\Phi(\tau(x)) \in A_{n-1}$ such that $\partial_{i} \Phi(\tau(x))=e_{n-2}$ for all $i$. These elements form a subgroup $\Gamma_{n-1} \subset A_{n-1}$. Two maps $\Phi_{0}$ and $\Phi_{1}$ are $\mu$-homotopic if there exists a function $\mu: S^{n} \rightarrow A$ satisfying (2.6.2), (2.6.3), (2.6.4). Such a function is completely determined by its value $\mu(x)$ on the unique nondegenerate simplex $x \in S^{n}$. (2.6.2) and (2.6.3) become:

$$
\begin{aligned}
& \partial_{0} \mu(x)=\Phi_{0}(\tau(x))^{-1} \Phi_{0}(\tau(x)), \\
& \partial_{i} \mu(x)=e_{n-1}, \quad i>0 .
\end{aligned}
$$

Let us recall Kan's combinatorial definition of homotopy groups. Let $A$ be a simplicial group and let $\Gamma_{n-1} \subset A_{n-1}$ be the subgroup of elements $a \in A_{n-1}$ such that $\partial_{i} a=0$ for all $i$. An element $a \in \Gamma_{n-1}$ is called null-homotopic if there exists $b \in A_{n}$ such that $\partial_{i} b=e_{n-1}$ for $i>0$ and $\partial_{0} b=a$. The subgroup $\gamma_{n-1} \subset \Gamma_{n-1}$, of null-homotopic elements is a normal subgroup and

$$
\pi_{n-1}(A)=\Gamma_{n-1} / \gamma_{n-1} \text {. }
$$

But (2.7.2) and (2.7.3) show that $\Phi_{0}$ and $\Phi_{1}$ are $\mu$-homotopic if and only if $\Phi_{0}(\tau(x))^{-1} \Phi_{1}(\tau(x)) \in \gamma_{n-1}$ so that we have a corollary of Theorem 2.6.5 the

THEOREM 2.7.4. The equivalence classes of principal cofibre bundles with cofibre $F S^{n}$ and cobase $A$ are in 1-1 correspondence with $\pi_{n-1}(A)$.

Theorem 2.7.4 is the dual of the following known result: The equivalence classes of principal fibre bundles over a space $X$ with fibre $K(Z, n)$ are in 1-1 correspondence with $H^{n+1}(X ; Z)$. 
3. Proof of Theorem 2.2.3. We shall prove by induction the following statement:

$P_{n}$ : For all $k \leqq n$ we can choose elements ${ }^{1} x \in G$ in $1-1$ correspondence with the canonical generators $x \in K$ of $F=F K$ such that:

$$
\begin{aligned}
s_{i} x & =s_{i}{ }^{1} x & & \text { for all } x \in K_{k}, k \leqq n-1, i \geqq 0 \\
\partial_{0}{ }^{1} x & =t(x) \partial_{0} x, & & t(x) \in A \text { for all } x \in K_{k}, k \leqq n \\
\partial_{i}{ }^{1} x & =\partial_{i} x & & \text { for all } x \in K_{k}, k \leqq n, i>0 \\
\Psi\left({ }^{1} x\right) & ={ }^{1} x^{\prime} x^{\prime \prime} . & &
\end{aligned}
$$

Assume that $P_{n-1}$ holds and let us prove $P_{n}$. If $x \in K_{n}, x=s_{i} y, y \in K_{n-1}$ take ${ }^{1} x=s_{i}{ }^{1} y$ so that $s_{i}{ }^{1} y=s_{i}{ }^{1} y$. Let $x \in K$ be nondegenerate and let $x^{*} \in G$ be such that $r_{2} \Psi: G \rightarrow F$ satisfies $\Psi\left(x^{*}\right)=x^{\prime *} x^{\prime \prime}$. Then,

$$
\Psi\left(\partial_{i} x^{*} \partial_{i}^{1} x^{-1}\right)=\partial_{i} x^{\prime *} \partial_{i} x^{\prime \prime} \partial_{i}\left(x^{\prime \prime}\right)^{-1} \partial_{i}^{1} x^{-1}
$$

and since $\partial_{i} x^{\prime \prime}=\left(\partial_{i} x\right)^{\prime \prime}, \Psi\left(\partial_{i} x^{*} \partial_{i}^{1} x^{-1}\right)=\partial_{i}\left(x^{\prime}\right)^{*} \partial_{i}^{1} x^{-1}, t_{i}^{*}(x)=\partial_{i} x^{*} \partial_{i}^{1} x^{-1}$ is an invariant element i.e. belongs to $A$ :

$$
\partial_{i} x^{*}=t_{i}^{*}(x) \partial_{i}^{1} x .
$$

Applying for $i>0, i<j$ the basic relations between faces to (3.1) we deduce

$$
\partial_{i} t_{j}^{*}(x)=\partial_{j-1} t_{i}^{*}(x), \quad i>0, i<j .
$$

Since $t_{i}^{*}(x) \in A$ and $A$ is a simplicial group we can apply to (3.2) Kan's extension condition and find $y \in A_{n}$ such that

$$
\partial_{i} y=t_{i}^{*}(x), \quad i>0 \text {. }
$$

Let ${ }^{1} x=y^{-1} x^{*}$. Then

$$
\Psi(x)=\Psi\left(y^{-1}\right) \Psi\left(x^{*}\right)=y^{-1}\left(x^{\prime}\right)^{*} x^{\prime \prime}={ }^{1} x^{\prime} x^{\prime \prime} .
$$

Further by (3.1) and (3.3), for $i>0$

$$
\begin{aligned}
& \partial_{i}{ }^{1} x=\left(\partial_{i} y\right)^{-1} \partial_{i} x^{*}=t_{i}^{*}(x)^{-1} t_{i}^{*}(x) \partial_{i}^{1} x=\partial_{i}^{1} x, \\
& \hat{o}_{0}{ }^{1} x=\left(\partial_{0} y\right)^{-1} t_{0}^{*}(x) \partial_{i}^{1} x=t(x) \partial_{i}^{1} x, \quad t(x) \in A .
\end{aligned}
$$

Thus, we have proved $P_{n}$ for nondegenerate simplexes. One can show that there is no ambiguity in the selection of representatives for degenerate simplexes i.e. that $z=s_{i} x=s_{j} y$ implies $s_{i}{ }^{1} x=s_{j}{ }^{1} y$, so that ${ }^{1} z$ can be chosen in a well-defined way.

It remains to prove that the degenerate elements satisfy $P_{n}$. For $i<0, i<j$

$$
\partial_{i} s_{j} x=\partial_{i} s_{j}^{1} x=s_{j-1} \partial_{i} x=s_{j-1} \partial_{i} x=\partial_{i} s_{j} x \text {. }
$$

For $i>0$,

$$
\begin{gathered}
\partial_{i} s_{i} x=\partial_{i} s_{i} x=x=\partial_{i} s_{i} x, \\
\partial_{i+1} s_{i} x=\partial_{i+1} s_{1}^{i} x={ }^{1} x=\partial_{i+1} s_{i} x .
\end{gathered}
$$

Finally for $i>j+1 \geqq 1$, by the inductive hypothesis

$$
\partial_{i} s_{j} x=\partial_{i} s_{j}^{1} x=s_{j} \partial_{i-1}^{1} x=s_{j} \partial_{i-1} x .
$$


We have proved by induction $P_{n}$ for all $n$. Call $F$ the subgroup of $G$ generated by the elements ${ }^{1} x, x \in K$. Then by Theorem 1.6.6, $G_{n}=A_{n} * F_{n}$ for all $n$ and $P_{n}$ shows, that for the corresponding simplicial groups we have a twisted free product decomposition $G=A *_{t} F$, with twisting function $t: K \rightarrow A$. This completes the proof of Theorem 2.2.3.

4. Proof of Theorem 2.4.1. We first prove that every twisting function $r: K \rightarrow B$ vanishes on elements of the form $s_{0} x$. By definition

$$
\partial_{0} s_{0}^{1} x=r\left(s_{0} x\right) \partial_{0}^{1} s_{0} x=r\left(s_{0} x\right)^{1} x .
$$

On the other hand,

$$
\partial_{0} s_{0}^{1} x=\partial_{0} s_{0}^{1} x={ }^{1} x .
$$

From (4.1) and (4.2) we obtain

$$
r\left(s_{0} x\right)=e_{n-1} \text {. }
$$

Let us define a function $\Phi$ on the free generators $\tau(x)$ of $G K$ by

$$
\Phi(\tau(x))=r(x)^{-1}
$$

If $x=s_{0} y$ then $\tau\left(s_{0} y\right)=e_{n-1}$ and by (4.3), $r(x)=e_{n-1}$. Thus $\Phi$ can be extended to a homomorphism $\Phi: G K \rightarrow B$, such that by (4.3)'

$$
r(x)=\Phi(\tau(x))^{-1}=\Phi(t(x)) .
$$

Let $G=A *_{t} F, F=F K$. Then by computation:

$$
\begin{gathered}
s_{i} t=t s_{i+1}, \quad i \geqq 0, \\
\partial_{0} t(x)=t \partial_{1}(x)\left(t \partial_{0}(x)\right)^{-1}, \quad \text { for all } x \in K . \\
\partial_{i} t(x)=t\left(\partial_{i+1} x\right), \quad i>0 .
\end{gathered}
$$

Lemma 4.8. The mapping $\Phi: G K \rightarrow B$ defined by (4.3) is a morphism in $\mathscr{G}$.

Proof. We have

$$
s_{i} \Phi(\tau(x))=\left(s_{i} r(x)\right)^{-1}=\Phi\left(\tau s_{i+1}(x)\right) .
$$

By the definition of $\tau, \tau s_{i+1}(x)=s_{i} \tau(x)$ so that

$$
s_{i} \Phi(\tau(x))=\Phi\left(s_{i} \tau(x)\right) .
$$

By (4.6) $\partial_{0} \Phi(\tau(x))=\Phi\left(\tau\left(\partial_{0} x\right)\right)^{-1} \tau\left(\partial_{1} x\right)$ and by definition $\tau\left(\partial_{0} x\right)^{-1} \tau\left(\partial_{1} x\right)=\partial_{0} \tau(x)$. Thus,

$$
\partial_{0} \Phi(\tau(x))=\Phi\left(\partial_{0} \tau(x)\right)
$$

By definition for $i>0, \partial_{i} \tau(x)=\tau\left(\partial_{i+1} x\right)$ and by (4.7) we obtain $\partial_{i} \Phi(\tau(x))=\Phi\left(\partial_{i} \tau(x)\right)$. The formulas (4.4), (4.9), (4.10) together with Theorem 2.3.1 establish Theorem 2.4.1. 
5. Appendix. Proof of the structure theorem for free co-operation.

The proof of Theorem 1.6.6 will follow by a thorough analysis of the structure of the comultiplication $\Phi$ and of the co-operation $\Psi$.

Structure of $\Phi: F \rightarrow F * F$ : We shall denote by $w^{\prime}$ the element $w \in F$ considered in the first factor, and by $w^{\prime \prime}$ the same element considered in the second factor.

$$
w=x_{1}^{\alpha} \cdots x_{p}^{\alpha}
$$

(where the $x_{i}^{\prime}$ 's are generators and $\alpha_{i} \in Z$ )

$$
\Phi(w)=\left(x_{1}^{\prime} x_{2}^{\prime \prime}\right)^{\alpha_{1}} \cdots\left(x_{p}^{\prime} x_{p}^{\prime \prime}\right)^{\alpha_{p}}
$$

where none of the $x_{i}^{\prime}$ and $x_{i}^{\prime \prime}$ cancel.

If $\alpha_{p}>0$, the last factor will be $x_{p}^{\prime \prime}$, if $\alpha_{p}<0$ the last factor will be $x_{p}^{\prime-1}$. In the expression of $(\Phi * 1) \circ \Phi: F \rightarrow F * F * F$, the element $w \in F$ will be denoted by $w^{\prime}$ if it belongs to the first factor and by $w^{\prime \prime}, w^{m}$ if it belongs to the second or third factor. The left hand $\Phi$ operates by the rule $\Phi\left(\left(x_{i}^{\prime}\right)\right)=x_{i}^{\prime} x_{i}^{\prime \prime}$ while the identity 1 operates by $1 w^{\prime \prime}=w^{\prime \prime}$. In the expression of $(1 * \Phi) \circ \Phi$, however, 1 operates by $1 w^{\prime}=w^{\prime}$ and the first $\Phi$ operates by $\Phi\left(x_{i}^{\prime \prime}\right)=x_{i}^{\prime \prime} x_{i}^{\prime \prime}$. Therefore

$$
\begin{aligned}
& (\Phi * 1) \circ \Phi(w)=\left(x_{1}^{\prime} x_{1}^{\prime \prime} x_{1}^{m}\right)^{\alpha_{1}} \cdots\left(x_{p}^{\prime} x_{p}^{\prime \prime} x_{p}^{m}\right)^{\alpha_{p}} \\
& (1 * \Phi) \circ \Phi\left(w^{\prime}\right)=\left(x_{1}^{\prime} x_{1}^{\prime \prime} x_{1}^{m}\right)^{\alpha_{1}} \cdots\left(x_{p}^{\prime} x_{p}^{\prime \prime} x_{p}^{m}\right)^{\alpha_{p}} .
\end{aligned}
$$

If $\alpha_{p}>0$, the last factor is $x_{p}^{m}$, if $\alpha_{p}<0$ the last factor is $x_{p}^{\prime \prime-1}$.

Let us consider a free co-operation $\Psi: G \rightarrow G * F$ of $(F, \Phi)$. We shall put in $G * F * F$ one prime on the elements of $G$, two primes on the elements of the first $F$, three primes on the elements of the second $F$. In the expression $(1 * \Phi) \circ \Psi$ : $G \rightarrow G * F * F, \Phi$ acts by the rule $\Phi\left(x_{i}^{\prime \prime}\right)=x_{i}^{\prime \prime} x_{i}^{\prime \prime}$, whereas $1 g^{\prime}=g^{\prime}$. In the expression $(\Psi * 1) \circ \Psi: G \rightarrow G * F * F, 1 w^{\prime \prime}=w$ whereas the first $\Psi$ on the left acts by the rule $\Psi\left(g^{\prime}\right)=\Psi(g)$.

Let for some $g \in G$,

$$
\begin{gathered}
\Psi(g)=g_{1}^{\prime} w_{1}^{\prime \prime} \cdots g_{n}^{\prime} w_{n}^{\prime \prime}, \quad g_{i} \in G, w_{i} \in F . \\
w_{n}=x_{1}^{\alpha} \cdots x_{p}^{\alpha}, .
\end{gathered}
$$

Case I. $w_{n}=e$. Then

$$
\begin{gathered}
\Psi(g)=g_{1}^{\prime} w_{1}^{\prime \prime} \cdots w_{n-1}^{\prime \prime} g_{n}^{\prime} \quad \text { where } w_{n-1}=x_{1}^{\beta_{1}} \cdots x_{q}^{\beta_{q}}, \quad B_{q} \neq 0 \\
(1 * \Phi) \circ \Psi(g)=g_{1}^{\prime} \Phi\left(w_{1}^{\prime \prime}\right) \cdots g_{n-1}^{\prime}\left(x_{1}^{\prime \prime} x_{1}^{\prime \prime}\right)^{\beta_{1}} \cdots\left(x_{q}^{\prime \prime} x_{q}\right)^{\beta_{q}} g_{n}^{\prime} \\
(\Psi * 1) \circ \Psi(g)=\Psi\left(g_{1}\right) w_{1} \cdots \Psi\left(g_{n-1}\right) x_{1}^{\beta_{1}} \cdots x_{q}^{\beta} \Psi\left(g_{n}\right)
\end{gathered}
$$

$\Psi\left(g_{n}\right) \in G * F$ and its expression contains only once and twice primed letters. For convenience, we shall call a segment (of a reduced word) any maximal subword containing letters with the same number of primes. Therefore,

Case $\mathrm{I}_{\mathrm{a}} . \beta_{q}>0$. The last segment of $(1 * \Phi) \circ \Psi(g)$ reduces to $g_{n}^{\prime}$, and the preceding letter is $x_{q}^{m \beta_{q}}$. The word $(\Psi * 1) \circ \Psi(g)$ ends with $\Psi\left(g_{n}\right)$ so that

$$
\left(\mathrm{I}_{\mathrm{a}}\right) \quad \Psi\left(g_{n}\right)=g_{n}^{\prime} .
$$


Case $\mathrm{I}_{\mathrm{b}} . \beta_{\mathrm{q}}<0$. The last two segments of $(1 * \Phi) \circ \Psi(g)$ not containing " $m$ " " are $x_{q}^{\prime-1} g_{n}^{\prime}$; this has to be equal to the corresponding part in $(\Psi * 1) \circ \Psi$ hence

Case II. In (5.4), $\alpha_{p} \neq 0$ :

$$
\left(\mathrm{I}_{\mathrm{b}}\right) \quad \Psi\left(g_{n}\right)=x_{q}^{\prime \prime-1} g_{n}^{\prime} .
$$

$$
\begin{aligned}
& (1 * \Phi) \circ \Psi(g)=g_{1}^{\prime} \Phi\left(w_{1}^{\prime \prime}\right) \cdots g_{n}\left(x_{1}^{m} x_{1}^{m}\right)^{\alpha_{1}} \cdots\left(x_{p}^{m} x_{p}^{m}\right)^{\alpha_{p}} \\
& (\Psi * 1) \circ \Psi(g)=\Psi\left(g_{1}\right) w_{1}^{m} \cdots \Psi\left(g_{n}\right) x_{1}^{m} \alpha_{1} \cdots x_{p}^{m \alpha_{p}} .
\end{aligned}
$$

Case $\mathrm{II}_{\mathrm{a}}, \alpha_{p}>0$. The last segment belonging to $F$ in (5.6) is $w_{n}^{m}=x_{1}^{m \alpha_{1}} \cdots x_{p}^{m \alpha_{p}}$ whereas in (5.6) this segment reduces to $x_{p}^{m}$. Thus in the Case $\mathrm{II}_{\mathrm{a}}, w_{n}=x_{p}$. Let again $w_{n-1}=x_{1}^{\beta_{1}} \cdots x_{q}^{\beta}, \beta_{q} \neq 0$.

Case $\mathrm{II}_{\mathrm{aa}} . \alpha_{p}>0, \beta_{q}>0$. We have already seen that $w_{n}=x_{\mathrm{p}}$ and (5.5) becomes

$$
(1 * \Phi) \circ \Psi(g)=g_{1}^{\prime} \Phi\left(w_{1}^{\prime \prime}\right) \cdots x_{2}^{m} g_{n}^{\prime} x_{p}^{\prime \prime} x_{p}^{m}
$$

whereas (5.6) becomes

$$
(\Psi * 1) \circ \Psi(g)=\Psi\left(g_{1}\right) w_{1}^{m} \cdots x_{q}^{m} \Psi\left(g_{n}\right) x_{p}^{m} .
$$

Comparing (5.7) and (5.8) we get

$$
\left(\mathrm{II}_{\mathrm{aa}}\right) \quad \Psi\left(g_{n}\right)=g_{n}^{\prime} x_{p}^{\prime \prime} .
$$

Case $\mathrm{II}_{\mathrm{ab}} \cdot \alpha_{p}>0, \beta_{q}>0$. (5.5) and (5.6) become, in this case,

$$
\begin{aligned}
& (1 * \Phi) \circ \Psi(g)=g_{1}^{\prime} \Phi\left(w_{1}^{\prime \prime}\right) \cdots x_{q}^{m-1} x_{q}^{\prime \prime-1} g_{n}^{\prime} x_{p}^{\prime \prime} x_{p} \\
& (\Psi * 1) \circ \Psi(g)=\Psi\left(g_{1}^{\prime}\right) w_{1}^{m} \cdots x_{q}^{m}\left(g_{n}\right) x_{p}^{m} .
\end{aligned}
$$

The last segments containing " " " and " "" in (5.9) and (5.10) are respectively $x_{q}^{\prime \prime} g_{n}^{\prime} x_{p}^{\prime \prime}$ and $\Psi\left(g_{n}\right)$ so that:

$$
\left(\mathrm{II}_{\mathrm{ab}}\right) \quad \Psi\left(g_{n}\right)=x_{q}^{\prime \prime-1} g_{n}^{\prime} x_{p}^{\prime \prime} .
$$

Case $\mathrm{II}_{\mathrm{b}} \cdot \alpha_{p}<0$. In (5.5), the last segments (with " "”) reduces to $x_{p}^{\prime \prime-1}$ whereas in (5.6) it is empty because (5.6) ends with $x_{p}^{m}$. Therefore case $\mathrm{II}_{\mathrm{b}}$ is impossible.

Let us compute $\Psi\left(g g_{n}^{-1}\right)$ in Case $\mathrm{I}_{\mathrm{a}}, \mathrm{I}_{\mathrm{b}}, \mathrm{II}_{\mathrm{aa}}, \mathrm{II}_{\mathrm{ab}}$

$$
\begin{aligned}
& \left(\mathrm{I}_{\mathrm{a}}\right): \quad \Psi(g)=g_{1}^{\prime} w_{1}^{\prime \prime} \cdots g_{n}^{\prime}, \quad \Psi\left(g_{n}^{-1}\right)=g_{n}^{\prime-1} \\
& \Psi\left(g g_{n}^{-1}\right)=g_{1}^{\prime} w_{1}^{\prime \prime} \cdots w_{n-1}^{\prime \prime}, \text { a shorter word. } \\
& \left(\mathrm{I}_{\mathrm{b}}\right): \quad \Psi(g)=g_{1}^{\prime} w_{1}^{\prime \prime} \cdots x_{q}^{\prime \prime-1} g_{n}^{\prime}, \quad \Psi\left(g_{n}^{-1}\right)=g_{n}^{\prime}{ }^{-1} x_{q}^{\prime \prime} \\
& \Psi\left(g g_{n}^{-1}\right)=g_{1}^{\prime} w_{1}^{\prime \prime} \cdots x_{q}^{\prime \prime-1} g_{n}^{\prime} g_{n}^{\prime-1} x_{q}^{\prime \prime} \text {, a shorter word. } \\
& \left(\mathrm{II}_{\mathrm{aa}}\right): \quad \Psi(g)=g_{1}^{\prime} w_{1}^{\prime \prime} \cdots g_{n}^{\prime} x_{p}^{\prime \prime}, \quad \Psi\left(g_{n}^{-1}\right)=x_{p}^{\prime \prime-1} g_{n}^{-1} \\
& \Psi\left(g g_{n}^{-1}\right)=g_{1}^{\prime} w_{1}^{\prime \prime} \cdots g_{n}^{\prime} x_{p}^{\prime \prime} x_{p}^{\prime \prime-1} g_{n}^{\prime-1}, \text { a shorter word. } \\
& \left(\mathrm{II}_{\mathrm{ab}}\right): \quad \Psi(g)=g_{1}^{\prime} w_{1}^{\prime \prime} \cdots x_{q}^{\prime \prime-1} g_{n}^{\prime} x_{p}^{\prime \prime}, \quad \Psi\left(g_{n}^{\prime-1}\right)=x_{p}^{\prime \prime-1} g_{n}^{\prime-1} x_{q}^{\prime \prime} \\
& \Psi\left(g g_{n}^{-1}\right)=g_{1}^{\prime} w_{1}^{\prime \prime} \cdots x_{q}^{\prime \prime}-1 g_{n}^{\prime} x_{p}^{\prime \prime} x_{p}^{\prime \prime}{ }^{-1} g_{n}^{\prime-1} x_{q}^{\prime \prime} \text {-again a shorter word. }
\end{aligned}
$$


We can prove by induction on the length of the word $\Psi(g)$, that $G$ is generated by elements $g$ which satisfy either $\left(\mathrm{I}_{\mathrm{a}}\right)$ or $\left(\mathrm{II}_{\mathrm{aa}}\right)$ or $\left(\mathrm{II}_{\mathrm{ab}}\right)$ (if $g$ satisfies $\left(\mathrm{I}_{\mathrm{b}}\right)$ then $g^{-1}$ satisfies $\left.\left(\mathrm{II}_{\mathrm{aa}}\right)\right)$. Hence,

LeMma 5.11. There exists a system of generators $g \in G$ such that either $\left(\mathrm{I}_{\mathrm{a}}\right)$ : $\Psi(g)=g^{\prime}$ or $\left(\mathrm{II}_{\mathrm{a}}\right): \Psi(g)=g^{\prime} x^{\prime \prime}$ where $x$ is a free generator of $F$, or $\left(\mathrm{II}_{\mathrm{ab}}\right): \Psi(g)=$ $x_{1}^{\prime \prime-1} g^{\prime} x_{2}^{\prime \prime}$ where $x_{1}, x_{2}$ are free generators of $F$.

We now prove

LEMMA 5.12. Let the co-operation of $F$ on $G$ be free. Then the generators of the type $\mathrm{II}_{\mathrm{ab}}$ can be eliminated, $G$ remaining generated by the generators of type $\mathrm{I}_{\mathrm{a}}$ and $\mathrm{II}_{\mathrm{aa}}$.

Proof. Let us call a generator $x \in F$ of the first kind, if $\Psi(g)=g^{\prime} x^{\prime \prime}$ for some $g \in G$, and of the second kind otherwise. If $\Psi(g)=\left(x^{\prime \prime}\right)^{\prime-1} g^{\prime} x^{\prime \prime}$ then $x^{\prime}$ and $x$ are of the same kind. For, let $x$ be of the first kind and $\Psi(h)=h^{\prime} x^{\prime \prime}$. Then $\Psi\left(h g^{-1}\right)=$ $\left((h g)^{\prime}\right)^{-1}\left(x^{\prime \prime}\right)^{\prime}$ and $x^{\prime}$ is also of the first kind. Let $g_{1}^{\prime} w_{1}^{\prime \prime} \cdots g_{n}^{\prime} w_{n}^{\prime \prime}=\Psi(g) \in G * H$. Then $r_{2} \Psi(g)=w_{1} \cdots w_{n}$, where $w_{1} \cdots w_{n}$ are words in $x_{i}$. The sum of all exponents of the generators of the second kind in $r_{2} \Psi(g)$ is even since these generators occur in pairs (they come from generators $g_{i} \in G$ satisfying $\mathrm{II}_{\mathrm{ab}}$ ). By hypothesis the cooperation is free i.e. $r_{2} \Psi^{*}: G \rightarrow F$ is an epimorphism. Suppose $x$ is of the second kind and $r_{2} \Psi(g)=x$, i.e. $w_{1} \cdots w_{n}=x$. Since all the generators of the second kind cancel out to $x$, their sum of exponents must be 1 , which contradicts the above assertion. Hence all generators are of the first kind. But then if $\Psi(g)=\left(x^{\prime \prime}\right)^{\prime-1} g^{\prime} x^{\prime \prime}$, where $x$ and $x^{\prime}$ are free generators of $F$, by the preceding remark, $x^{\prime}$ must be a generator of the first kind and there exists an $h \in G, \Psi(h)=h^{\prime}\left(x^{\prime \prime}\right)^{\prime}$. Thus $g=h\left(h^{-1} g\right)$ where $\Psi\left(h^{-1} g\right)=h^{\prime-1} g^{\prime} x^{\prime \prime}$. Therefore $G$ is generated by elements $g$ such that either $\Psi(g)=g^{\prime}$ or $\Psi(g)=g^{\prime} x^{\prime \prime}$, where $x$ is a free generator of $F$.

For each generator $x_{i} \in F$, let ${ }^{1} x_{i} \in G$ be such that $\Psi\left({ }^{1} x_{i}\right)={ }^{1} x_{i}^{\prime} x_{i}^{\prime \prime}$ and let ${ }^{1} F$ be the subgroup of $G$ generated by the ${ }^{1} x_{i}$ 's.

LEMMA 5.13. The mapping $r_{2} \Psi{ }^{1} F:{ }^{1} F \rightarrow F$ is an isomorphism.

Proof. Clearly, $r_{2} \Psi$ maps ${ }^{1} F$ onto $F$. Let $g={ }^{1} x_{1}^{\alpha} 1 \cdots{ }^{1} x_{n}^{\alpha}$. Then $r_{2}(g)=x_{1}^{\alpha} \cdots \cdots x_{n}^{\alpha}$ $=e$ if and only if the word $g$ is identically trivial.

Let $A$ be the group of elements invariant under $\Psi$ i.e. such that $\Psi(g)=g^{\prime}$. Then if $\Psi(g)=g^{\prime} x_{i}^{\prime \prime}$ and ${ }^{1} x_{i} \in{ }^{1} F, \Psi\left({ }^{1} x_{i}\right)={ }^{1} x^{\prime} x_{i}^{\prime \prime}$ so that $\Psi\left(g x_{i}^{-1}\right)=g^{\prime}\left({ }^{1} x_{i}^{\prime}\right)^{-1}$ and $g^{1} x_{i}^{-1} \in A$. Hence by Lemma 5.12, $G$ is generated by $A$ and ${ }^{1} F$. In order to complete the proof of the theorem it is enough to show that $G=A *{ }^{1} F . A *^{1} F$ is constituted of reduced words $a_{1}{ }^{1} w_{1} \cdots a_{n}{ }^{1} w_{n}$ where $a_{i} \in A$ and ${ }^{1} w_{i}={ }^{1} x_{1}^{\alpha_{1}^{1}} \ldots{ }^{1} x_{n_{i}^{n}}^{\alpha^{1}}$, where only $a_{1}$ and ${ }^{1} w_{n}$ may equal $e$.

There is a canonical epimorphism $\pi: A * F \rightarrow G$ where $\pi(a)=a, \pi\left({ }^{1} w\right)=w$. We have to show that if $W=a_{1}{ }^{1} w_{1} \cdots a_{n}{ }^{1} w_{n} \in A *{ }^{1} F$ is a reduced word and $g=\pi(W)$ $=e$, then $W=e$ i.e. that $\pi$ is an isomorphism.

Case I. ${ }^{1} w_{n}=e$. If $n=1, W=a_{1}$ and $g=a_{1}=e$ so that $W=e$. If $n \geqq 2$ let ${ }^{1} w_{n-1}=$ ${ }^{1} x_{1}^{\alpha}{ }^{\alpha} \cdots{ }^{1} x_{p}^{\alpha}$ where $\alpha_{p} \neq 0$. The last part of $\Psi^{\prime}(g)$ is $\cdots\left({ }^{1} x_{1}^{\prime} x_{1}^{\prime \prime}\right)^{\alpha_{1}} \cdots\left({ }^{1} x_{p}^{\prime} x_{p}^{\prime \prime}\right)^{\alpha_{p}} a_{n}^{\prime}$. 
Case $\mathrm{I}_{\mathrm{a}}: n \geqq 2, \alpha_{p}>0$. Then the representation of $\Psi(g)=e$ ends with $x_{p}^{\prime \prime} a_{n}^{\prime}, a_{n} \neq e$ which is a contradiction. Hence in this case $n \geqq 2$ is contradictory and if $n=1$, $W=e$.

Case $\mathrm{I}_{\mathrm{b}}: n \geqq 2, \alpha_{p}<0$. The representation of $\Psi(g)$ ends with $x_{p}^{\prime \prime-1}\left({ }^{1} x_{p}^{\prime}\right)^{-1} a_{n}^{\prime}$ and $\Psi(g)=e$ implies ${ }^{1} x_{p}^{-1} a_{n}^{-1}=e$ which is impossible since $a_{n} \in A,{ }^{1} x_{p} \notin A$. Again contradiction.

Case II. $e \neq w_{n}={ }^{1} x_{1}^{\alpha}{ }^{1}{ }^{1} x_{p}^{\alpha} p$.

Case $\mathrm{II}_{\mathrm{a}}: \alpha_{p}>0$. The representation of $\Psi(g)=e$ ends with ${ }^{1} x_{p}^{\prime} x_{p}^{\prime \prime}$ which is a contradiction.

Case $\mathrm{II}_{\mathrm{b}}: \alpha_{p}<0$. The representation of $\Psi(g)=e$ ends with $\cdots x_{p}^{\prime \prime-1}\left({ }^{1} x_{p}^{\prime}\right)^{-1}$ which again is a contradiction.

Therefore $\pi(W)=e$ implies $W=e$ and $\pi: A *^{1} F \simeq G$. This completes the proof of Theorem 1.6.6.

\section{BIBLIOGRAPHY}

1. M. G. Barrat, V. K. A. M. Gugenheim and J. C. Moore, On semi-simplicial fibre bundles, Amer. J. Math. 81 (1959), 639-657.

2. H. Cartan and J.-P. Serre, Espaces fibres et groupes d'homotopie, C. R. Acad. Sci. Paris 234 (1952), 288-290.

3. B. Eckmann and P. J. Hilton, Groupes d'homotopie et dualité. I, II, III, C. R. Acad. Sci. Paris 246 (1958), pp. 244, 2555, 2993.

4. - Structure maps in group theory, Fund. Math. 50 (1961), 207-221.

5. —, Group-like structures in general categories, I, Math. Ann. 145 (1962), 227-255; II, Math. Ann. 151 (1963), 150-186.

6. S. Eilenberg and J. A. Zilber, Semi-simplicial complexes and singular homology, Ann. of Math. 51 (1950), 499-513.

7. P. J. Hilton, The fundamental group as a functor, Bull. Soc. Math. Belg. 14 (1962), 153-177.

8. - Homotopy and duality, Lecture Notes, Cornell University, Ithaca, N. Y., 1959.

9. D. M. Kan, A combinatorial definition of homotopy groups, Ann. of Math. 67 (1958), 282-312.

10. - On homotopy theory and c.s.s. groups, Ann. of Math. 68 (1958), 38-53.

11. - On monoids and their dual, Bol. Soc. Mat. Mexicana 3 (1958), 52-61.

12. - Minimal free c.s.s. groups, Illinois J. Math. 2 (1958), 537-547.

13. P. J. May, Simplicial objects in algebraic topology, Van Nostrand, Princeton, N. J., 1967.

14. J. Milnor, The construction FK, Lecture notes, Princeton University, Princeton, N. J., 1956.

15. - The geometric realization of a semi-simplicial complex, Ann. of Math. 65 (1957), 357-362.

16. J. C. Moore, Seminar on algebraic homotopy theory, Mimeographed notes, Princeton University, Princeton, N. J., 1956.

17. - Semi-simplicial complexes and Postnikov systems, Sympos. Intern. de Topologia Algebraica, Mexico, 1956.

MCGILl UNIVERSTTY, Montreal, Canada

UNIVERSITÉ DE MONTRÉAL, Montréal, CANADa 